УДК 621.391 .812

\title{
ЭФФЕКТИВНОСТЬ НОВЫХ ВЕРСИЙ СFАR ОБНАРУЖИТЕЛЕЙ ПРИ ОБРАБОТКЕ М-КОРРЕЛИРОВАННЫХ ИМПУЛЬСОВ В УСЛОВИЯХ ПОМЕХ
}

\author{
МОХАМЕД Б.ЭЛЬ МАШАД \\ Университет аль-Азхар, \\ Ezunem, Kaup, Насер-Сити
}

\begin{abstract}
Аннотация. Обнаружение движущейся цели MTI (moving target indicator) на фоне помех представляет собой одну из наиболее важных задач радиолокационной системы. Для решения этой задачи необходимо подавить или устранить помехи, добиваясь при этом как можно меньшего ослабления полезного сигнала. В этом отношении радиолокационная станция МТI способна обнаруживать движущуюся цель при наличии помех. Радар MTI представляет большой интерес для гражданского и военного применения, где он снижает помехи, отраженные от стационарного или медленно перемещающегося объекта. Кроме того, чтобы принимать решения о наличии цели, обработка МТI может применяться с автоматическим обнаружением. B этой ситуации CFAR обнаружитель - это общий адаптивный алгоритм, используемый в радиолокационных системах для обнаружения отраженного от цели сигнала на фоне шума и помех. Однако наличие МТI усложняет анализ производительности системы обнаружения, поскольку ее выходная последовательность коррелируется, даже если ее входная последовательность может быть некоррелированной. Цель этой статьи - проанализировать производительность процессора радиолокационных сигналов, который состоит из нерекурсивного MTI, за которым следует квадратичный интегратор и цепь CFAR обнаружителя новой версии, работа которого основана на гибридной комбинации алгоритмов СА и ТМ. Производительность процессора оценивается для случая, когда окружающая среда считается идеальной (однородной), а также в случае наличия сигнала от ложной цели в опорных ячейках. Численные результаты показали, что производительность процессора улучшается или за счет увеличения количества некогерентно интегрированных импульсов, или за счет уменьшения корреляции между последовательными импульсами, при условии, что вероятность ложной тревоги поддерживается постоянной.
\end{abstract}

Ключевые слова: процессор CFAR; индикатор движущейся цели; MTI; помеха; производный детектор; некогерентное интегрирование М импульсов; наличие нескольких целей

\section{1. ВВЕДЕНИЕ}

Основная задача первичных радаров, используемых для управления воздушным движением или движением судов, заключается в обнаружении всех целей в зоне наблюдения и, если воздушное судно обнаружено, РЛС определяет пеленг, дальность, скорость и т.д. Следовательно, обнаружение представляет собой фундаментальную задачу, на основе которой радиолокационный приемник решает продолжить или прекратить свою обработку.
В реальных ситуациях помехи - это сложный случайный пространственно-временной процесс. Это главная проблема для работы радаров вне помещения, и нет никаких стандартных мер по ее устранению. Природа помех зависит от параметров системы и радиолокатоpa, поскольку существует большое количество абонентов и чрезмерно используется спектр. В связи с этим электромагнитные помехи являются обычным явлением в современных устройствах связи и электронном оборудовании. 


\section{БИБЛИОГРАФИЧЕСКИЙ СПИСОК}

1. Dillard, G. M.; Rickard, J. T. "Performance of an MTI followed by incoherent integration for nonfluctuating signals," Proc. of IEEE Int. Radar Conf., 28-30 Apr. 1980, San Diego, USA. IEEE, 1980, pp. 194-199.

2. El Mashade, M. B. "Performance analysis of the OS family of CFAR schemes with incoherent integration of M-pulses in the presence of interferers," IEE Proc. Radar, Sonar Navig., Vol. 145, No. 3, pp. 181-190, June 1998. DOI: 10.1049 /ip-rsn: 19981607.

3. Jose Raul Machado-Fernandez, Norelys Mojena-Hernandez, "Evaluation of CFAR detectors 
performance", ITECKNE, Vol. 14, No. 2, December 2017 , pp. $170-178$.

4. El Mashade, M. B. "M-correlated sweeps performance analysis of mean-level CFAR processors in multiple target environments," IEEE Trans. Aerospace Electronic Systems, Vol. 38, No. 2, p. 354-366, Apr. 2002. DOI: 10.1109/TAES.2002.1008971.

5. Md. Maynul Islam, Mohammed Hossam-E-Haider, "Detection Capability and CFAR Loss Under Fluctuating Targets of Different Swerling Model for Various Gamma Parameters in RADAR", International Journal of Advanced Computer Science and Applications, Vol. 9, No. 2, 2018, pp.90-93.

6. El Mashade, M. B. "M-correlated sweeps performance analysis of adaptive detection of radar targets in interference-saturated environments," Ann. Telecommun., Vol. 66, No. 11-12, p. 617-634, 2011. DOI: 10.1007/s12243-010-0236-5.

7. Wang, W. Q. Radar Systems: Technology, Principles and Applications. Nova Science Publishers, Inc, 2013.

8. Yadav, Ajay Kumar; Kant, Laxmi. "Moving target detection using VI-CFAR algorithm on MATLAB platform," Int. J. Advanced Research Computer Science and Software Engineering, Vol. 3, No. 12, p. 915-918, Dec. 2013.

9. Chang J. Kim, "A new formula to predict the exact detection probability of a generalized order statistics CFAR detector for a correlated Rayleigh target ", ETRI journal, Vol. 16, No. 2, July 1994, pp.15-25.
10. Ivković, Dejan; Andrić, Milenko; Zrnić, Bojan. "A new model of CFAR detector," Frequenz, Vol. 68, Nos. 3-4, p. 125-136, Mar. 2014. DOI: 10.1515/freq-2013$\underline{0087}$.

11. Mandal, Amritakar; Mishra, Rajesh. "An adaptive clutter suppression technique for moving target detector in pulse Doppler radar," Radioengineering, Vol. 23, No. 1, p. 84-95, Apr. 2014. URI: https://www.radioeng.cz/ fulltexts/2014/14 $01 \quad 0084$ 0095.pdf.

12. EYuhua Qin, Huili Gong, Ting Liu, "A New CFAR Detector based on Automatic Censoring Cell Averaging and Cell Averaging", TELKOMNIKA, Vol. 11, No. 6, June 2013, pp. 3298-3303.

13. Shome, Subhankar; Bera, Rabindra Nath; Sur, Samarendra Nath; Adhikary, Rabi. "Moving target detection and Doppler extraction using digital spread spectrum radar," Int. J. Intelligent Systems Applications, Vol. 6, No. 10, p. 47-54, 2014. DOI: 10.5815/ijisa.2014. 10.07 .

14. El Mashade, M. B. "Heterogeneous performance evaluation of sophisticated versions of CFAR detection schemes," Radioelectron. Commun. Syst., Vol. 59, No. 12, p. 536-551, 2016. DOI: 10.3103/S0735272716120025.

15. El Mashade, M. B. "Heterogeneous performance analysis of the new model of CFAR detectors for partially-correlated $\chi 2$-targets," J. Systems Engineering Electronics, Vol. 29, No. 1, p. 1-17, Feb. 2018. DOI: 10.21629/JSEE.2018.01.01. 